\title{
Diseño de un ciclo de mejora en la enseñanza de la asignatura "Matemáticas". Experiencia en el aula
}

\author{
INMACULADA CONCEPCIÓN MASERO MORENO \\ Universidad de Sevilla \\ Dpto de Economía Aplicada III \\ imasero@us.es \\ ORCID: https://orcid.org/0000-0002-4023-8916 \\ D.O.I.: http://dx.doi.org/10.12795/JDU.2018.i01.52 \\ Pp.: 922-938
}

\section{Resumen:}

El ciclo de mejora que se expone en este trabajo ha sido el resultado de un proceso de reflexión y análisis sobre la necesidad e importancia del estudio de las funciones reales de variable real y las derivadas en el contexto económico, empresarial y financiero. Su diseño se configura en torno a un mapa de contenidos, un modelo metodológico y una secuencia de actividades. Este ciclo ha sido llevado al aula en la asignatura Matemáticas del doble grado en Finanzas y Contabilidad y Relaciones Laborales y Recursos Humanos. En el trabajo se recoge dicha experiencia y se comentan los resultados obtenidos.

Palabras claves: Matemáticas, Doble grado en Finanzas y Contabilidad y Relaciones Laborales y Recursos Humanos, docencia universitaria, experimentación docente universitaria, aprendizaje activo 


\section{Contexto}

El ciclo de mejora del curso anterior 2017/2018 se realizó en la asignatura cuatrimestral Matemáticas de primer curso del Doble Grado en Finanzas y Contabilidad y Relaciones Laborales y Recursos Humanos de la Universidad de Sevilla. El nuevo ciclo se realizará sobre esta misma asignatura, que tiene asignado 6 créditos y pertenece a la formación básica del Grado en Finanzas y Contabilidad.

En el presente curso se ha reorganizado el temario, aunque su contenido se mantiene. En esta nueva organización, el tema que se abordaba en el ciclo del curso anterior se ha organizado en dos temas diferentes y no consecutivos temporalmente. Esta circunstancia hace que el nuevo ciclo de mejora no pueda ser una extensión del anterior. Sin embargo, sí ha sido la base sobre la que se ha construido un nuevo ciclo sobre el primer tema del presente curso. En este tema se aborda, de forma más extensa y con mayor profundidad, la materia de la primera parte del ciclo anterior, el Análisis de las funciones reales de variable real. Los contenidos son las funciones reales de una variable real elementales, la continuidad y las derivadas y se completa con su relación y uso en las funciones económicas a través de las funciones marginales y la elasticidad. El nuevo ciclo se propone para ser realizado durante las dos primeras semanas de clase.

El contexto es muy similar al del curso pasado. Se mantiene un único grupo y la docencia está organizada en dos bloques semanales de dos horas, jueves y viernes por la tarde, siempre en el mismo espacio. Hay que señalar que dicho espacio está organizado en una única columna de bancas fijas (seis por fila) que, además está pegada a una de las paredes. Esta disposición dificulta la realización del trabajo en grupo de forma cómoda para el alumnado y me impide llegar a los tres estudiantes que están sentados en las bancas próximas a la pared para comprobar lo que están haciendo o bien mantener un dialogo sobre su trabajo. 
Por lo tanto, en este caso, el escenario en el que se va a realizar este ciclo no es apropiado para favorecer la interacción entre estudiantes y docente.

El número de alumnos matriculados asciende a 61. Mayoritariamente proceden del Bachillerato de Ciencias Sociales.

\section{Diseño del Ciclo de Mejora Docente}

En esta asignatura es muy importante introducir desde el punto de vista económico el tema que se va a trabajar. Para evitar la mera transmisión de contenidos se plantea esta parte desde la necesidad de responder determinadas preguntas tanto económicas como de contenido matemático y la relación entre ambas.

El objetivo de la asignatura es:

"proporcionar los conceptos y herramientas matemáticas necesarias para abordar el estudio y análisis de fenómenos económicos básicos y garantizar que el alumnado las domina con soltura".

De este objetivo se deduce la importancia que tiene el conseguir un nivel de dominio adecuado de los conceptos del tema del ciclo de mejora, como son las funciones y las derivadas. Parte del alumnado conoce su estudio y cálculo y lo puede desarrollar con mayor o menor destreza. Sin embargo, hay que dejar constancia de un hecho importante: al iniciar el curso, el alumno no tiene por qué tener conocimiento de funciones económicas, aunque sean básicas. Y, además, los conceptos que aquí se proponen como aplicación directa no se explican en otras asignaturas de forma paralela.

\section{Mapa de contenidos}

\section{El mapa de contenidos gira en torno al siguiente obje- tivo central:}




\section{y de los siguientes subojetivos:}

1. Mostrar cómo se utiliza el concepto de función real en los modelos económicos básicos

2. Aplicar el concepto de derivada en los modelos económicos básicos.

Las preguntas que guían y conectan los contenidos del mapa conceptual del tema elegido para el ciclo de mejora son:

P1. ¿Existe relación entre la demanda y el precio? ¿Cómo se puede expresar matemáticamente?

P2. ¿La relación existe para cualquier valor del precio? Es decir, ¿cualquier precio tiene asociada una demanda?

P3. ¿Podría el precio estar relacionado y condicionado por la demanda? ¿Cómo lo expresaría matemáticamente?

P4. ¿Cómo se podría calcular la demanda total de una empresa que produce dos tipos de refrescos de cola si conocemos la demanda de cada uno de ellos?

P5. ¿Cómo podríamos calcular el ingreso de una empresa?

P6. ¿Cómo podríamos calcular el beneficio de una empresa?

P7. ¿Cómo podemos expresar la demanda de un bien si su precio varía en el tiempo?

P8. ¿Cómo se puede calcular la variación de la demanda de un bien cuando el precio pasa del valor $\mathrm{P}$ al valor $\mathrm{P}^{\prime}$ ?

P9. ¿Cómo se puede calcular matemáticamente cómo afecta a la demanda la variación del precio en una unidad?

P10. ¿Cómo se puede calcular matemáticamente cómo afecta a la demanda la variación del precio eliminando las unidades de medidas de ambos?

A partir de estas, los contenidos se conectan en el mapa como aparece recogido en la figura siguiente: 
FUNCIONES REALES

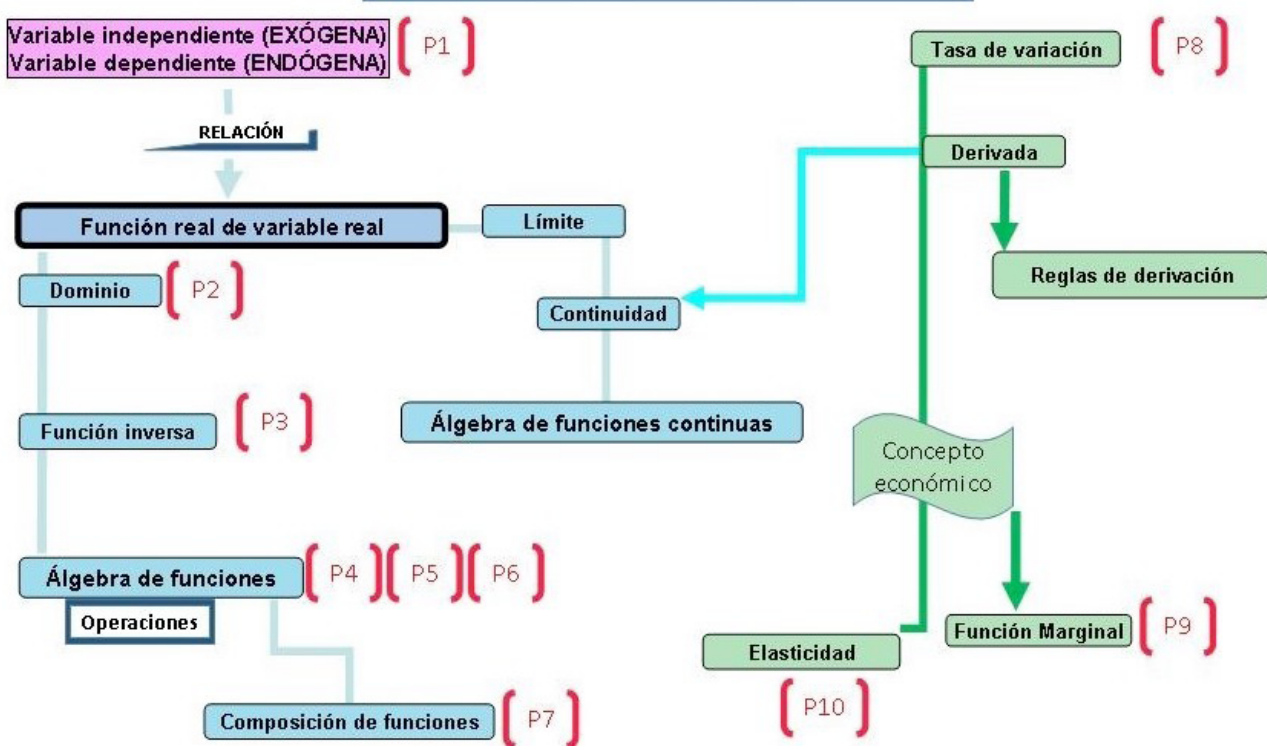

Figura 1. Mapa de conceptual y preguntas

Las preguntas que guían el mapa son las que integran el cuestionario inicial-final que me permitirá evaluar el conocimiento. Este cuestionario no se centra en la parte mecánica del cálculo de los conceptos, sino en comprobar si el alumnado entiende su significado y, más allá de su simple cálculo, es capaz de interpretar su resultado. Esto puede permitir, a posteriori, realizar algunas preguntas antes de abordar su aplicación económica para comprobar si son capaces de relacionar conceptos económicos con conceptos matemáticos.

\section{Modelo metodológico posible}

El modelo metodológico en el que se apoyó el ciclo del curso pasado creo que resultó adecuado para el tema, permitiéndome desarrollar bien la enseñanza de la materia. Por estas razones voy a mantener su estructura básica durante este ciclo. 
El modelo se configura en torno a tres bloques centrales (figura 2) que se abordan desde un perfil teórico y/o práctico (también se ha indicado el bloque de inicio de cada clase). El tamaño de la figura que representa a cada bloque está relacionado con el tiempo de duración de cada parte de la clase (elipses). También aparecen recogidas las tareas de cada bloque (rectángulos de la derecha) como, por ejemplo, la aclaración de dudas.

La estructura de este modelo está recogida en la figura 2.

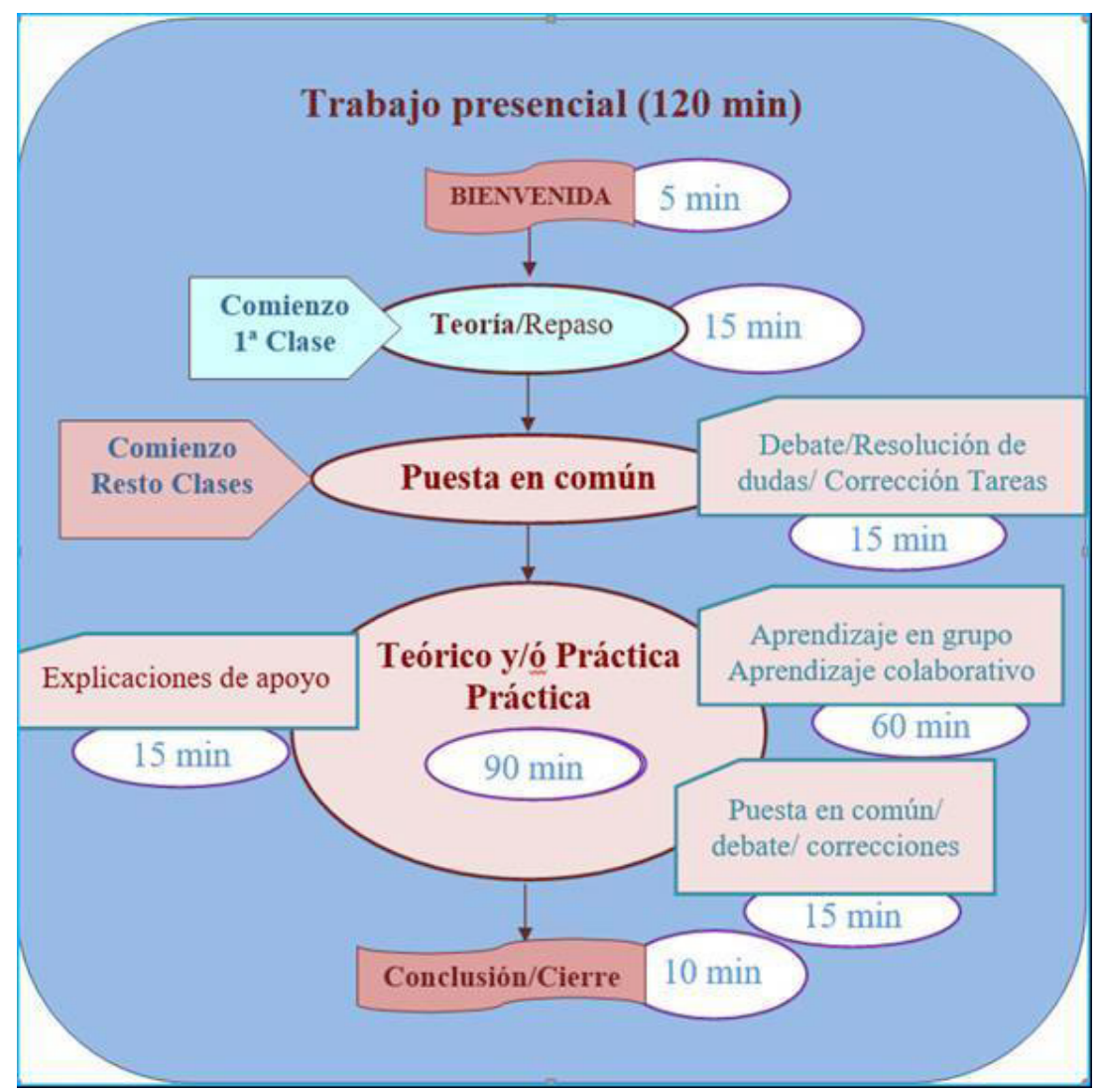

Figura 2. Modelo didáctico

Las clases se organizan en cuatro bloques claramente diferenciados: Jornadas de Formación e Innovación Docente del Profesorado I № 1 (2018)
Esta obra se distribuye con la licencia Creative Commons Reconocimiento-NoComercial-SinObraDerivada Internacional (CC BY-NC-ND 4.0.) 
1. Cada clase comienza con una bienvenida (5 minutos).

2. El segundo bloque está organizado de la forma siguiente.

- En la primera clase se realiza el cuestionario inicial y se introduce el tema. En dicha introducción se aborda la importancia de las Matemáticas en la Economía aludiendo a los primeros economistas que introdujeron su uso en el siglo XVIII.

- A partir de la segunda clase, este tiempo se dedica a resolver las dudas que surgen a partir del trabajo no presencial. Atendiendo al tipo de dudas, es el propio grupo el que participa de forma activa aclarando dichas dudas.

3. El tercer bloque ocupa la mayor parte de la clase, 90 minutos, y durante éste, el alumnado se organiza en grupos. El trabajo se estructura en dos partes:

- En la primera, los grupos resuelven los ejercicios, tanto teóricos como prácticos, propuestos en el boletín de ejercicios o por mí, para comprobar que los conceptos matemáticos trabajados en las clases anteriores han quedado perfectamente asimilados. Junto a estos, se introducen otros conceptos nuevos para empezar a trabarlos. Como apoyo yo utilizaré presentaciones en Power Point y aclaraciones en la pizarra. Los grupos exponen la resolución de los ejercicios y problemas.

- Una vez comprobado que los conceptos matemáticos han sido asimilados y se manejan con soltura, propongo un problema de enunciado económico para su resolución (en total son doce problemas).

- Para finalizar se realiza la actividad de contraste correspondiente. 
4. En cada sesión se finaliza con un resumen del trabajo realizado y se concreta el trabajo no presencial para la siguiente clase (por ejemplo, diferentes lecturas). En la última sesión se vuelve a proponer el cuestionario inicial para responder.

Para el trabajo no presencial propuesto en este último bloque, el alumnado puede encontrar las lecturas en la plataforma de enseñanza virtual de la Universidad. Además, dispone de material monográfico para su consulta.

\section{Secuencia de actividades}

Las actividades de cada sesión se plantean para trabajar en grupo y consisten en resolver:

- ejercicios prácticos (pertenecientes al boletín de la asignatura) para ejercitar el cálculo de los conceptos matemáticos de dominio, límite continuidad y derivadas

- y problemas de enunciado económico (del boletín de la asignatura y propuestos por Barrios y otros, 2005) en los que se aborda la aplicación de los conceptos matemáticos a través del cálculo de la marginalidad y la elasticidad, aplicando la derivada e interpretando los resultados.

A continuación, en la siguiente tabla parece desglosada la propuesta de actividades para cada una de las cuatro clases que integran el ciclo de mejora. 
Tabla 1

Secuencia de actividades

\begin{tabular}{|c|c|c|}
\hline Clase & $\begin{array}{l}\text { Ejercicios prácticos } \\
\text { Boletín de la asignatura }\end{array}$ & $\begin{array}{l}\text { Problemas contextualizados } \\
\text { (Barrios y otros, 2005) }\end{array}$ \\
\hline Primera & 1 (dominios) & $\begin{array}{l}\text { Problema 1: ejemplo } 2.1 \text { (pp.43-44) } \\
\text { Problema 2: ejemplo } 2.7 b \text { (pp.53) }\end{array}$ \\
\hline Segunda & 2 (continuidad) & $\begin{array}{l}\text { Problema 3: ejemplo } 2.3 \text { (pp.45-46) } \\
\text { Problema 4: ejemplo 2.3b (pp.45) } \\
\text { Problema 5: ejemplo } 2.31 \\
\text { (pp.114-118) }\end{array}$ \\
\hline Tercera & 3 (derivadas) & $\begin{array}{l}\text { Problema 6: estudio de una lec- } \\
\text { tura (Funciones relacionadas con } \\
\text { la función } \mathrm{y}=\mathrm{f}(\mathrm{x}) \text {, de uso genera- } \\
\text { lizado en el campo de la Ciencia } \\
\text { Económica y Empresarial (pp. 111- } \\
\text { 113) y elaboración de un esquema } \\
\text { a partir de esta }\end{array}$ \\
\hline Cuarta & $\begin{array}{l}4 \text { (marginalidad) } \\
5 \text { (elasticidad) }\end{array}$ & $\begin{array}{l}\text { Problema 7: ejemplo 2.23b (pp.82) } \\
\text { Problema 8: ejemplo 2.23d } \\
\text { (pp.82-83) } \\
\text { Problema 9: ejemplo 2.27b } \\
\text { (pp.93-94) } \\
\text { Problema 10, } 11 \text { y 12: ejemplos } \\
\text { (pp.114-118) }\end{array}$ \\
\hline
\end{tabular}

Las lecturas propuestas para cada clase pertenecen al libro de Barrios y otros (2005) y al de Aguado y Hamoudi (2014). La elección de ambos se debe a que el primero sitúa las Matemáticas en el contexto económico-empresarial y el segundo introduce los conceptos económicos y alude en determinadas ocasiones al comportamiento de las funciones económicas a través de la representación gráfica de las funciones.

He planificado dos pruebas de control a través de cuestionarios de respuesta múltiple. He decidido probar con esta alternativa porque en clase ya estamos trabajando con problemas de perfil económico, y esta opción me permite introducir cambios que pueden dinamizar el aprendizaje y comprobar si se interpretan las relaciones entre los diferentes conceptos y sus valores.

La programación inicial ha sido la siguiente: 
- Primera prueba: en el ecuador del tema, al finalizar la segunda clase. Así, el alumnado ha tenido tiempo de asimilar y comprender los conceptos básicos económicos en los que se utilizan las funciones y, lo que esto implica para su estudio (8 preguntas).

- Segunda prueba: al finalizar el ciclo y antes del cuestionario final (9 preguntas).

\section{Aplicación del ciclo de mejora}

El ciclo se ha realizado durante las cuatro primeras clases del curso y ha sido completado por 43 estudiantes.

Me ha parecido conveniente realizar un cuestionario de conocimientos básicos el día de la presentación de la asignatura, para determinar el nivel de conocimiento matemático previo en relación con el tema de las funciones reales de variable real. Las respuestas me han permitido hacer un perfil, individual y grupal, tanto del nivel de conocimiento como del manejo de los conceptos matemáticos básicos. También este día indiqué a los estudiantes que en la plataforma estaban disponibles las lecturas para cada clase del ciclo, indicando la importancia de su lectura y posterior reflexión antes de la clase.

A continuación, haré un recorrido por los aspectos más importantes del desarrollo del ciclo en el aula, indicando tanto los elementos del diseño expuesto que he mantenido como aquellos en los que ha sido necesario introducir algún cambio.

El primer día de clase he propuesto y realizado el cuestionario inicial con las 10 preguntas ya expuestas.

He seguido optando, al igual que el curso pasado con el primer ciclo que llevé al aula, por el aprendizaje activo en grupos colaborativos basado en problemas para el desarrollo de la parte central de cada clase. Esta combinación de metodologías ha permitido que los estudiantes construyan el conocimiento a partir del trabajo con las lecturas previas y con los problemas de cada sesión. 
Como ya he comentado, el escenario no favorece que el trabajo se desarrolle de forma cómoda para el alumnado ni para mí. He alternado el número de integrantes de los grupos, dos o tres estudiantes, de una clase a otra. Durante el trabajo en grupo, el alumnado se ha responsabilizado del aprendizaje y que ha implicado en el desarrollo de la clase.

Además de las lecturas, he propuesto al alumnado que intente los ejercicios del boletín antes de clase, ya que se trata de conceptos que conocen, aunque la profundidad de conocimiento y el nivel de dominio de los mismos es heterogéneo. Esto ha permitido que durante el trabajo en grupo se haya podido generar un debate, y posterior reflexión, sobre la resolución de estos ejercicios, enriqueciendo el desarrollo del proceso de aprendizaje.

Mi papel durante el trabajo de los grupos ha sido atender las consultas, supervisar el desarrollo que realizan los grupos que evidencian dificultades en el proceso e indicar con algunas ayudas la forma de continuar o terminar la resolución tanto de los ejercicios como de los problemas contextualizados.

Respecto a los grupos más aventajados, les he propuesto otros ejercicios diferentes a los ya propuestos, en los que tuvieran que relacionar las ideas y conceptos que ya comprenden y cuyo cálculo dominan. En estos grupos, los integrantes han afianzando y ampliado su aprendizaje y esta estrategia me ha permitido atender a la diversidad en el aula.

En determinadas ocasiones, he propuesto un debate en el conjunto de toda la clase para intentar aclarar algún punto problemático para la inmensa mayoría. También se han tratado estos puntos al resolver los problemas en la pizarra por alguno de los grupos.

Para evaluar el trabajo diario y motivar hacia el aprendizaje, los problemas propuestos se realizan como tareas de aprendizaje que, además, puedo utilizar como tareas de 
evaluación. Así, he podido comprobar si los alumnos han comprendido los conceptos y aprendido la materia.

En algunos conceptos más complejos he tenido que introducir en la clase tareas para corregir las deficiencias del proceso de aprendizaje y también afianzar lo aprendido. Por esta razón, he creído conveniente introducir un cambio en el planteamiento de las pruebas de control. He decidido hacer una única prueba de control con 10 preguntas que he seleccionado del total de las 17 que había elaborado. Para tomar esta decisión también me he apoyado en el hecho de que en cada clase he podido comprobar la evolución y los avances.

En el resultado de la prueba destaca que 37 de los 43 estudiantes han respondido correctamente al menos al $50 \%$ de las preguntas. Una vez finalizada, se ha corregido en el aula para generar un feedback inmediato con el alumnado. Los resultados atendiendo a la calificación obtenida por los estudiantes están recogidos en la tabla siguiente.

Tabla 2

Calificaciones de los alumnos en la prueba de control

\begin{tabular}{|l|l|l|l|l|l|l|l|}
\hline \% respuestas correctas & $\mathbf{9 0 \%}$ & $\mathbf{8 0} \%$ & $\mathbf{7 0 \%}$ & $\mathbf{6 0 \%}$ & $\mathbf{5 0 \%}$ & $\mathbf{4 0 \%}$ & $<=\mathbf{3 0 \%}$ \\
\hline Número estudiantes & 1 & 7 & 10 & 11 & 8 & 3 & 3 \\
\hline
\end{tabular}

El $86 \%$ de los estudiantes han superado la prueba, lo que implica que el alumnado ha comprendido los conceptos. Solo dos de las 10 preguntas han sido respondidas correctamente por un $28 \%$ y el $30 \%$, respectivamente. La primera hace referencia a la función inversa y su representación gráfica en el contexto económico y la segunda al significado de la marginalidad. Con esta información, he vuelto a insistir en estos puntos antes de cerrar el tema y realizar de nuevo el cuestionario inicial, que ahora pasa a ser cuestionario final.

Las preguntas del cuestionario inicial-final están relacionadas con las preguntas de la prueba de control. En 
particular, las preguntas 3 y 9 del cuestionario inicial-final hacen referencia a la función inversa y a la marginalidad, respectivamente. Para comprobar si las aclaraciones realizadas sobre estos dos temas, una vez corregida la prueba de control, han resuelto las dudas en torno a estos dos temas, voy a analizar las respuestas a la pregunta 3 y 9 del cuestionario inicial-final.

En ambos casos, he agrupado las respuestas atendiendo al razonamiento en términos económicos y/o matemáticos, resultando cinco grupos. Los dos primeros escalones, "No sabe/ No contesta" y "Sí/No" no implican ningún proceso de reflexión. En el tercer escalón aparece el razonamiento exclusivamente en términos económicos y en el cuarto escalón se justifica lo económico en términos matemáticos. En el último escalón he encuadrado las respuestas en las que además de razonar económica y matemáticamente, se relacionan estas dos perspectivas.

Para indicar cuáles son los porcentajes correspondientes al cuestionario final, los he enmarcado en un rectángulo. Las escaleras de aprendizaje para las dos preguntas aparecen desglosadas en las siguientes figuras.

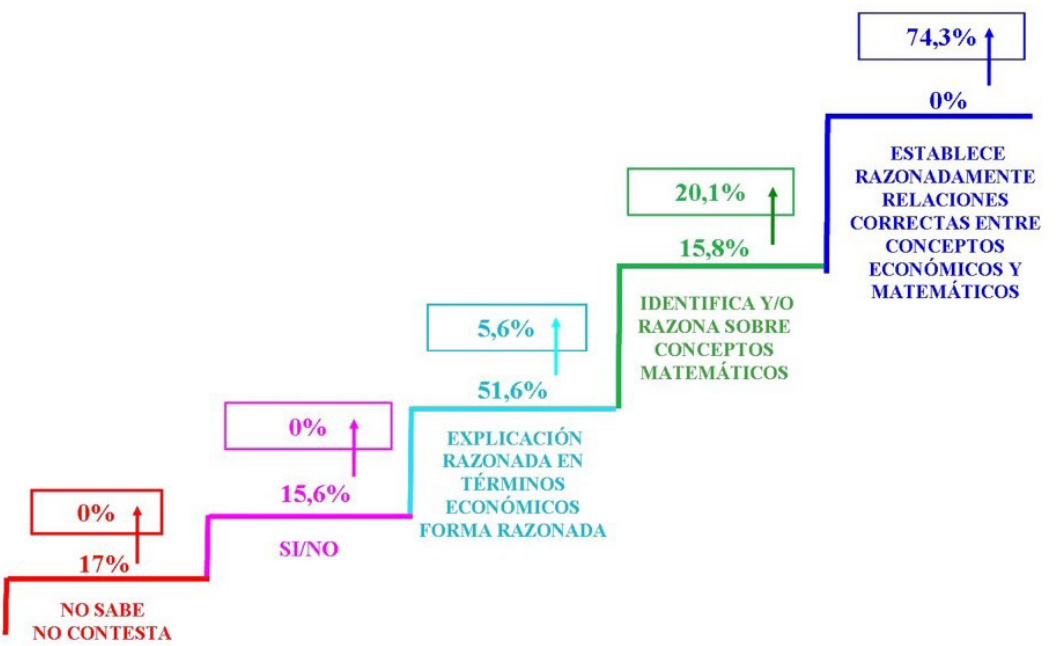

Figura 3. Escalera pregunta 3 del cuestionario inicial-final: ¿Podría el precio estar relacionado y condicionado por la demanda? ¿Cómo lo expresaría matemáticamente? 


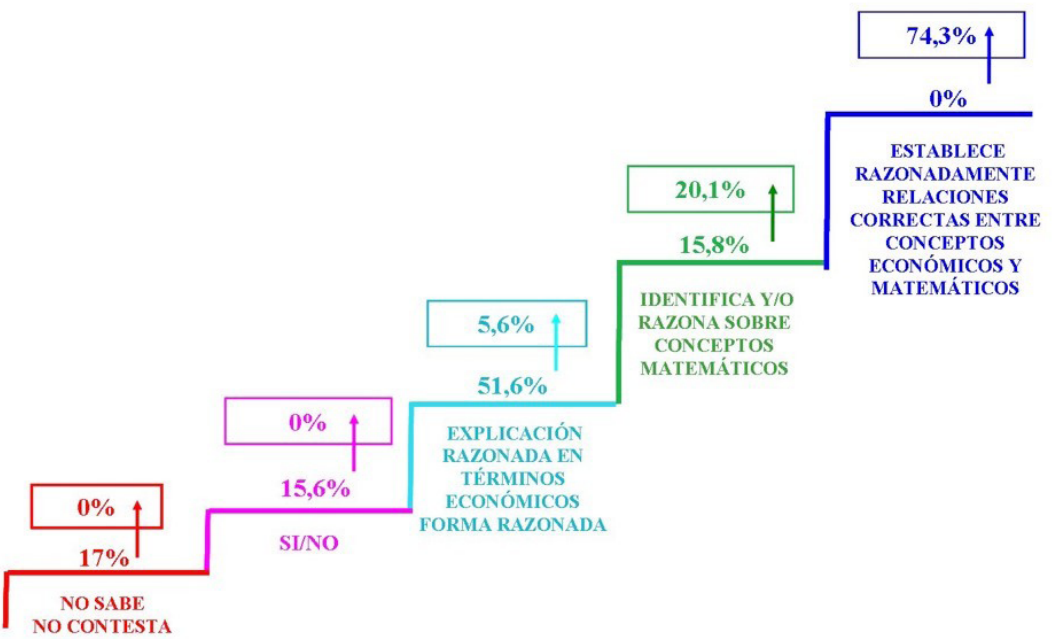

Figura 4. Escalera pregunta 9 del cuestionario inicial-final: ¿Podría el precio estar relacionado y condicionado por la demanda? ¿Cómo lo expresaría matemáticamente?

Si se observan los porcentajes correspondientes al cuestionario inicial (porcentajes de los que parten las flechas) en la escalera de cada pregunta, se comprueba que son pocos los estudiantes capaces de dar una respuesta perteneciente a los escalones 3 y 4 , y ninguno en el escalón más alto. Posiblemente esto se deba a que todavía no había habido toma de contacto con este tipo conceptos económicos al realizarse el cuestionario inicial en la primera clase.

Respecto al cuestionario final, queda patente el porcentaje de estudiantes que ha respondido a ambas preguntas con una perspectiva más amplia, incorporando los conceptos trabajados y apoyándose en las aclaraciones realizadas a raíz de los resultados de la prueba de control. Además, en ambas escaleras se confirma un avance en el aprendizaje de los dos puntos más problemáticos del tema, al no haber respuestas en los escalones inferiores.

Estos resultados indican que el hecho de resolver dudas e incidir en los conceptos problemáticos atendiendo a los resultados de la prueba de control incide favorablemente en el aprendizaje. 


\section{Evaluación del ciclo de mejora}

Comenzaré la evaluación del ciclo haciendo referencia a los resultados de la prueba de control y del cuestionario inicial-final que he expuesto en el apartado anterior, de los que se puede deducir que esta propuesta metodológica ha sido adecuada para la enseñanza de los contenidos correspondientes al análisis de las funciones reales de variable real.

Para poder evaluar el desarrollo del ciclo de mejora una vez finalizado, voy a utilizar el diario de clase. En este he recogido observaciones referentes a los siguientes puntos:

- el desarrollo del proceso de enseñanza como el número de alumnos que acuden a cada clase

- el cumplimiento de los objetivos previstos en referencia a la realización de las actividades programadas

- los conceptos que han resultado más problemáticos en cada sesión para proponer tareas no presenciales para reforzar su aprendizaje.

Según estas observaciones, el proceso se ha desarrollado de la forma programada sin ningún tipo de problema. Sin embargo, he recogido algunas sugerencias referentes al tiempo dedicado a algunos conceptos que me permitirá reajustar el tiempo dedicado a estos para el cronograma del próximo curso. Además, en base a la identificación de los conceptos más problemáticos de comprender por el alumno, voy a elaborar actividades de aprendizaje para intentar solventar estas dificultades desde el principio.

En todas las sesiones he intentado mantener un diálogo informal con los grupos de trabajo para poder comprobar si era necesario realizar ajustes en el contenido de las actividades en relación con su nivel de dificultad y el tiempo previsto para cada una de ellas. También, me ha servido para establecer una comunicación más cercana y fluida con los alumnos y comprobar si estaban cómodos con esta forma de trabajar en el aula y de aprender. 
Por último, he pedido al alumnado que me indicara por escrito cómo había sido la experiencia con el ciclo de mejora y que me indicaran las sugerencias que creyeran oportunas. De esta forma, el alumnado también evalúa el proceso en el que ha participado de forma activa y me permite detectar las deficiencias que éste puede percibir.

La opinión sobre la experiencia, la dinámica de las clases y las lecturas para preparar la clase es positiva. Aunque reconocen que se han adaptado bien al planteamiento propuesto, algunos estudiantes insisten en que les gustaría alguna clase tradicional dedicada en su totalidad a corregir ejercicios y problemas en la pizarra. Aqui hay que señalar que el ciclo se ha desarrollado en las primeras clases, lo que ha podido suponer un choque para estos estudiantes y evidentemente un esfuerzo tanto e el trabajo presencial como no presencial.

Partiendo de los resultados en las escaleras de las dos preguntas analizadas, comentados en el apartado anterior, me planteo programar la realización de este tipo de pruebas de control en futuros ciclos.

Una ventaja clara de realizar este ciclo de mejora al principio de curso es la posibilidad de situar, desde el primer momento, al alumnado como parte activa de una forma diferente de trabajar en el aula y conseguir que se adapte antes a este planteamiento.

El modelo metodológico ha dado buenos resultado a nivel de aprendizaje y de valoración por el alumnado. Creo que esto también ha generado confianza en el alumnado, sobretodo a la hora de trabajar en grupo.

Personalmente, me ha permitido ampliar mi visión sobre la enseñanza de los conceptos de este tema y las posibilidades de proponer su aprendizaje desde su justificación y necesidad dentro de este tipo de estudios. Si se mantiene el temario, en el próximo curso me planteo ampliar el ciclo al siguiente tema que aborda el Cálculo Integral. 
Por último, esta experiencia me ha ayudado como docente a seguir replanteándome la enseñanza que desarrollo en el aula y cómo propongo al alumnado que desarrolle su aprendizaje. Reflexionar sobre estos aspectos tan importantes creo que nos enriquece como docentes y nos ayuda a encontrar vías para mejorar nuestra docencia.

\section{Referencias bibliográficas.}

Aguado y Hamoudi (2014). Guía para comprender la microeconomía. Madrid: Pirámide.

Barrios, J.A., Campillo, M., Gil, M.C., González, C. y Pestano, C. (2005). Análisis de funciones en Economía y Empresa. Un enfoque multidisciplinar. Madrid: Díaz de los Santos.

Jornadas de Formación e Innovación Docente del Profesorado | № 1 (2018) Esta obra se distribuye con la licencia Creative Commons 